\title{
Isoliquiritigenin inhibits proliferation and induces apoptosis of U87 human glioma cells in vitro
}

\author{
GUO-SHENG ZHOU, LAI-JUN SONG and BO YANG
}

Neurosurgery Department, The First Affiliated Hospital of Zhengzhou University, Zhengzhou, Henan 450052, P.R. China

Received July 16, 2012; Accepted November 9, 2012

DOI: $10.3892 / \mathrm{mmr} .2012 .1218$

\begin{abstract}
Isoliquiritigenin (ISL), a member of the flavonoids, has been demonstrated to possess antitumor activity in various cancer cell lines in vitro and in vivo. In this study, we investigated the antitumor effects of ISL on U87 glioma cells in vitro. As determined by MTT assay, ISL inhibited the proliferation of U87 cells in a time-dependent and dose-dependent manner. The results of fluorescence-activated cell sorting (FACS) analysis suggested that ISL induced the apoptosis of the U87 cells and blocked cell cycle progression at the $\mathrm{S}$ and G2/M phases. Moreover, it was identified that ISL induced the apoptosis of the U87 cells in a caspase-dependent manner. Although treatment with the pan-caspase inhibitor Z-VAD-FMK efficiently blocked the ISL-induced caspase activation, it did not eliminate the ISL-induced cell death. Further examination using western blot analysis revealed that ISL upregulated p21/WAF1 and p27. These results indicate that cell cycle arrest and the caspase-mediated apoptosis pathway may participate in the antiproliferative activity of ISL in U87 cells by regulating the expression of specific molecules.
\end{abstract}

\section{Introduction}

Glioblastoma (GBM) is one of most common malignant brain tumors in adults (1). The incidence of GBM in China is also increasing (2). Despite developments in neurosurgery, chemotherapy and radiation therapy, the prognosis of GBM has not changed significantly in the last decade. Surgical resection is the most effective method of therapy for GBM, however the high frequency of tumor recurrence is the major obstacle for GBM clinical therapy (3). As the current treatment modalities of GBM are not satisfactory, new agents for potential use in GBM clinical therapy have attracted attention.

Herbal therapies are gradually being recognized and adopted in clinical cancer therapy, and traditional Chinese

Correspondence to: Professor Lai-Jun Song, Neurosurgery Department, The First Affiliated Hospital of Zhengzhou University, 1 East Jianshe Street, Zhengzhou, Henan 450052, P.R. China

E-mail: ljsong_zzu@163.com

Key words: isoliquiritigenin, glioma, apoptosis, cell cycle, caspase medicine has demonstrated effectiveness in cancer therapy (4-6), not only by attenuating the side-effects of radical or chemical therapy but also by exhibiting antitumor efficacy, inhibiting the recurrence and metastasis of cancer, improving the quality of life and aiding the recovery of patients.

Flavonoids are polyphenolic compounds which exist in certain types of green vegetables, fruits and other botanicals. The significant physiological functions of flavonoids are attracting an increasing amount of attention. The results of an epidemiological survey have demonstrated that the intake of vegetables and fruits with high flavonoid contents reduces the incidence of cancer, including lung cancer and colon cancer (7). As flavonoids have varied bioactivities, efficient antitumor activity and low toxicity, the development of flavonoids is of significant value in cancer therapy and prevention (8).

Isoliquiritigenin (ISL) is a flavonoid compound with a variety of bioactivities that is isolated from licorice (a legume) and shallot (a liliaceae), which are widely used in traditional Chinese medicine. The antitumor efficacy of ISL against various types of cancer has previously been demonstrated in vitro and in vivo (9-11). In the current study, the antitumor activity of ISL on the glioma cell line U87 was examined.

\section{Materials and methods}

Chemicals. ISL (Fig. 1A) was purchased from Sigma (St. Louis, MO, USA). It was dissolved in dimethyl sulfoxide (DMSO) and diluted prior to use. The pan-caspase inhibitor Z-VAD-FMK was purchased from Beyotime Institute of Biotechnology (Jiangsu, China). Antibodies against p21, p27, caspase-3 and $\beta$-actin were purchased from Santa Cruz Biotechnology (Santa Cruz, CA, USA). Horseradish peroxidase (HRP)-conjugated goat anti-mouse or anti-rabbit IgG were obtained from Beyotime Institute of Biotechnology. RPMI-1640 medium and fetal bovine serum (FBS) were supplied by Gibco (Grand Island, NY, USA). All other chemicals used in this study were biologically pure.

Cell culture and drug treatment. The U87 human glioma cells (American Type Culture Collection, ATCC; Manassas, VA, USA) were cultured in RPMI-1640 medium supplemented with $10 \% \mathrm{FBS}, 100 \mathrm{U} / \mathrm{ml}$ penicillin and $100 \mu \mathrm{g} / \mathrm{ml}$ streptomycin at $37^{\circ} \mathrm{C}$ in a humidified atmosphere containing $5 \% \mathrm{CO}_{2}$.

After the cells were grown to sub-confluence, they were pre-treated with various concentrations of ISL $(10-80 \mu \mathrm{M})$ or 
A

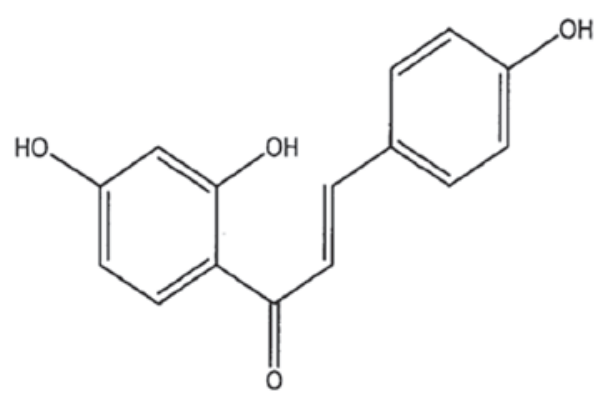

B

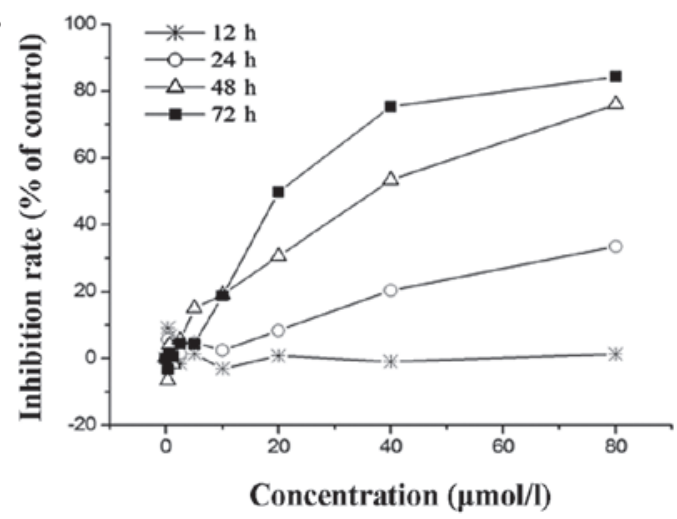

Figure 1. Growth inhibitory effect of isoliquiritigenin (ISL) on U87 glioma cells. (A) Chemical structure of ISL. (B) Time- and dose-dependent effects of ISL on the proliferation of U87 cells. Values represent the mean of three independent experiments.

pan-caspase inhibitor Z-VAD-FMK $(25 \mu \mathrm{M})$ in RPMI-1640 medium with $10 \%$ FBS for different times. Thereafter, the cells were washed twice with PBS and then cultivated for further assay.

Cell proliferation assay. U87 cells were plated in triplicate in 96-well plates (Corning Inc., Corning, NY, USA) at a density of 5,000 cells/well. Following overnight cultivation, various concentrations of ISL were added and cultivation of the cells was continued for 12, 24, 48 and $72 \mathrm{~h}$. The effects on cell growth were determined by methyl-thiazolyltetrazolium (MTT) assay. Briefly, $20 \mu \mathrm{l}$ MTT solution $(5 \mathrm{mg} / \mathrm{ml})$ was added to each well and the cells were incubated for another $4 \mathrm{~h}$ at $37^{\circ} \mathrm{C}$. Subsequently, $150 \mu \mathrm{l} \mathrm{DMSO}$ was added to dissolve the MTT formazan and the absorbance was measured using a microplate reader (Thermo Scientific, Rockford, IL, USA) at a wavelength of $570 \mathrm{~nm}$.

Cell cycle analysis. U87 cells were plated at a density of $1 \times 10^{6}$ cells $/ \mathrm{ml}$ medium in $50-\mathrm{mm}$ diameter dishes. ISL was added after $24 \mathrm{~h}$ of cultivation. The cells were harvested from the culture dishes $24 \mathrm{~h}$ later and stained with $70 \%$ ethanol at $-2^{\circ} \mathrm{C}$. Prior to FACS analysis, the cells were washed with PBS twice, resuspended and then incubated with $200 \mu \mathrm{g} / \mathrm{ml}$ RNase A (Sigma) for $60 \mathrm{~min}$ at $37^{\circ} \mathrm{C}$. Propidium iodide (PI; Sigma) was added at $50 \mu \mathrm{g} / \mathrm{ml}$ and the cells were incubated in the dark for 15-30 min. The samples were analyzed using a fluorescence-activated cell sorter (BD Calibur; BD Biosciences, San Jose, CA, USA).

FITC-Annexin V/PI apoptosis assay. U87 cells were plated at a density of $1 \times 10^{6}$ cells $/ \mathrm{ml}$ medium in $50-\mathrm{mm}$ diameter dishes. ISL was added after $24 \mathrm{~h}$ of cultivation. The cells were harvested from the culture dishes $24 \mathrm{~h}$ later by trypsinization. After washing twice with PBS, the cells were resuspended in $500 \mu 1$ binding buffer. Then, $5 \mu 1$ FITC-labeled Annexin V (Kaiji Biotechnology Ltd., Nanjing, China) and $5 \mu 1$ PI were added. Upon incubation in the dark for $15 \mathrm{~min}$ at room temperature, the samples were analyzed with a FACScan instrument (BD Calibur).

Preparation of cell lysates. Following treatment with ISL for $24 \mathrm{~h}$, the U87 cells were washed twice with ice-cold PBS and then scraped and lysed with RIPA lysis buffer (50 $\mathrm{nM}$ Tris, $\mathrm{pH}$ 7.4, $150 \mathrm{mM} \mathrm{NaCl}, 1 \%$ Triton X-100, $1 \%$ sodium deoxycholate, $0.1 \%$ SDS and $0.05 \mathrm{mM}$ EDTA). A cocktail of protein inhibitor mix $\left(25 \mathrm{mM} \mathrm{NaF}, 1 \mathrm{mM} \mathrm{Na}_{3} \mathrm{VO}_{4}, 20 \mathrm{mM}\right.$ $\beta$-glycerophosphate, $20 \mathrm{mM}$ PNPP, $1 \mathrm{mM}$ PMSF, $1 \mathrm{mg} / \mathrm{ml}$ leupeptin, $1 \mathrm{mg} / \mathrm{ml}$ aprotinin and $1 \mathrm{mg} / \mathrm{ml}$ pepstatin A) was also added to the RIPA lysis buffer. The samples were then centrifuged at $12,000 \times \mathrm{g}$ for $30 \mathrm{~min}$ at $4^{\circ} \mathrm{C}$ and the supernatants were collected as the total cell extracts. Protein concentrations were quantified using a BCA protein assay kit (Pierce, Rockford, IL, USA)

Western blot analysis. Western blot analysis was used to detect p21, p27 and caspase-3, with $\beta$-actin as a control. An aliquot of cell lysate containing $50 \mu \mathrm{g}$ protein was separated on $12 \%$ sodium dodecyl sulfate-polyacrylamide gel (SDS-PAGE), and then transferred to a $0.45-\mu \mathrm{m}$ polyvinylidene fluoride membrane. The membranes were blocked with $5 \%$ non-fat milk in Tris- $\mathrm{HCl}, \mathrm{pH} 7.5$, and $150 \mathrm{mmol} / \mathrm{l} \mathrm{NaCl}$ containing $0.1 \%$ Tween-20 for $1 \mathrm{~h}$ and then incubated with individual primary antibodies overnight at $4{ }^{\circ} \mathrm{C}$. The blots were then incubated with HRP-conjugated antibody. Signals were detected using enhanced chemiluminescence reagents (Millipore, Billerica, MA, USA) and densitometric analysis was performed with the use of X-ray film. The expression levels were normalized to $\beta$-actin and the control levels were set to $100 \%$.

Statistical analysis. The results in this study are expressed as the mean \pm SD. Treatment effects were compared using the Student's t-test and $\mathrm{P}<0.05$ was considered to indicate a statistically significant result.

\section{Results}

Inhibitory effects of ISL on the proliferation of glioma cells. The inhibitory effects of ISL on U87 glioma cells were assessed by MTT assay. The U87 cells were treated with various concentrations of ISL for different times and then examined by MTT assay. As shown in Fig. 1B, incubation with ISL for $12 \mathrm{~h}$ at concentrations of $0-80 \mu \mathrm{mol} / 1$ had no effect on the viability of the U87 cells. Otherwise, the inhibitory effects of ISL on the U87 cells increased as the time of treatment increased. 

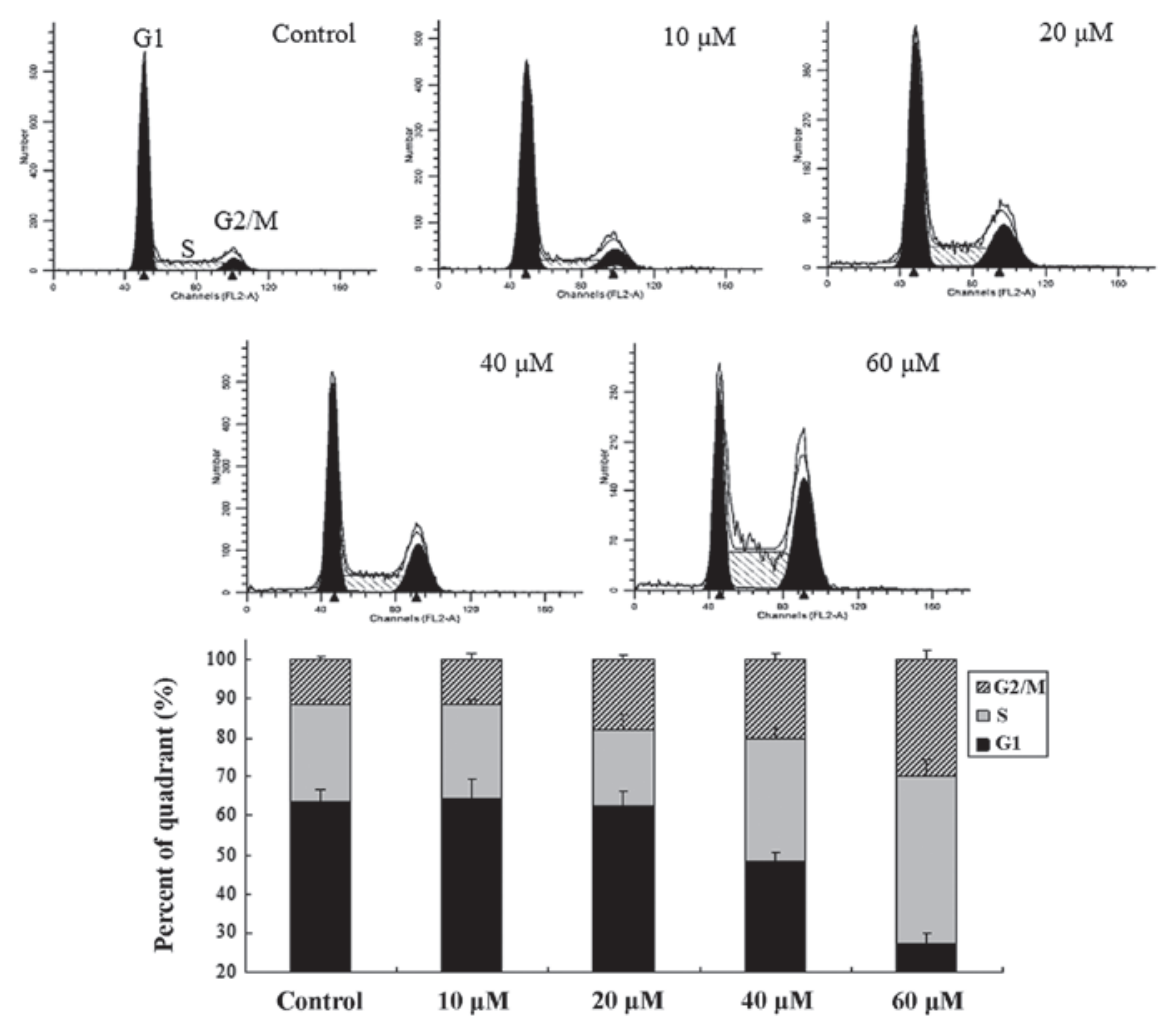

Figure 2. Effect of isoliquiritigenin (ISL) on the cell cycle of U87 cells. The U87 cells were exposed to various concentrations of ISL for $24 \mathrm{~h}$ and stained with propidium iodide to determine the DNA content. The percentages of the cells in each phase of the cell cycle were determined by flow cytometric analysis.

Cell cycle arrest induced by ISL. The effect of ISL on the cell cycle of the U87 cells was determined by FACS analysis. As shown in Fig. 2, ISL markedly induced $\mathrm{S}$ and G2/M phase arrest in a concentration-dependent manner. ISL at a concentration of $10 \mu \mathrm{M}$ had no evident effect on cell cycle arrest. The levels of $\mathrm{S}$ and $\mathrm{G} 2 / \mathrm{M}$ arrest increased as the ISL concentration increased. As the ISL concentration increased from 0 to $60 \mu \mathrm{mol} / \mathrm{l}$, an increase in the percentage of cells in the $\mathrm{G} 2 / \mathrm{M}$ phase from 11.55 to $29.91 \%$ and an increase in the percentage of cells in the S phase from 24.87 to $42.62 \%$ was observed. By contrast, the percentage of cells in the G1 phase decreased from 63.58 to $24.48 \%$ following treatment with ISL at corresponding doses.

Apoptosis induced by ISL. The apoptosis of the U87 cells induced by ISL was analyzed by FACS. Annexin V/PI analysis was used to examine the apoptosis of the U87 cells after $24 \mathrm{~h}$ of culturing in the presence of various concentrations of ISL. The results (Fig. 3) revealed that ISL induced apoptosis and necrosis of the U87 cells. As the ISL concentration increased, the percentages of apoptosis and necrosis were increased dose-dependently. As shown in Fig. 3, as the ISL concentration increased from 0 to $60 \mu \mathrm{M}$, the percentages of UR (secondary apoptotic or late stage necrotic), LR (early apoptotic) and UL (non-apoptotic necrotic) cells increased from 1.54 to $11.12 \%, 1.42$ to $8.76 \%$ and 7.77 to $16.93 \%$, respectively. As observed from Fig. 3, the percentages of UR and LR cells did not markedly change as the ISL concentration was increased from 40 to $60 \mu \mathrm{M}$, however the percentage of UL cells increased as the ISL concentration increased, which indicated that ISL was able to induce U87 cell death by a non-apoptotic process.

Apoptosis is induced by ISL via apoptosis-dependent and non-apoptosis-dependent pathways. To investigate the detailed mechanism of the ISL-induced apoptosis, the pan-caspase inhibitor Z-VAD-FMK was added and FACS was used to detect the change of cell apoptosis. Notably, the results of FACS (Fig. 4) revealed that the pan-caspase inhibitor Z-VAD-FMK decreased the ISL-induced apoptotic cell death (LR and UR), however, the percentages of the U87 glioma cells undergoing necrotic cell death (UL) were not affected by the addition of the pan-caspase inhibitor. This indicates that ISL was able to induce the death of U87 cells via a non-apoptotic pathway. As shown in Fig. 4, the treatment with Z-VAD-FMK did not alter the percentage of living cells. The results of western blot using caspase-3 antibody (Fig. 5) verified that treatment with ISL induced the activation of caspase-3, and that the pan-caspase inhibitor Z-VAD-FMK at $25 \mathrm{M}$ was able to block the activation of caspase- 3 and thereby reduce the percentage of early apoptotic cells. However, the percentage of total living cells was not altered by the treatment with Z-VAD-FMK, and the percentage of nonapoptotic necrotic cells was increased along with the ISL concentration.

Effects of ISL on p27 and p21 expression levels determined by western blot analysis. To further investigate the mechanism of the ISL-induced cell cycle arrest and apoptosis, the protein expression levels of p21 and p27 were determined 

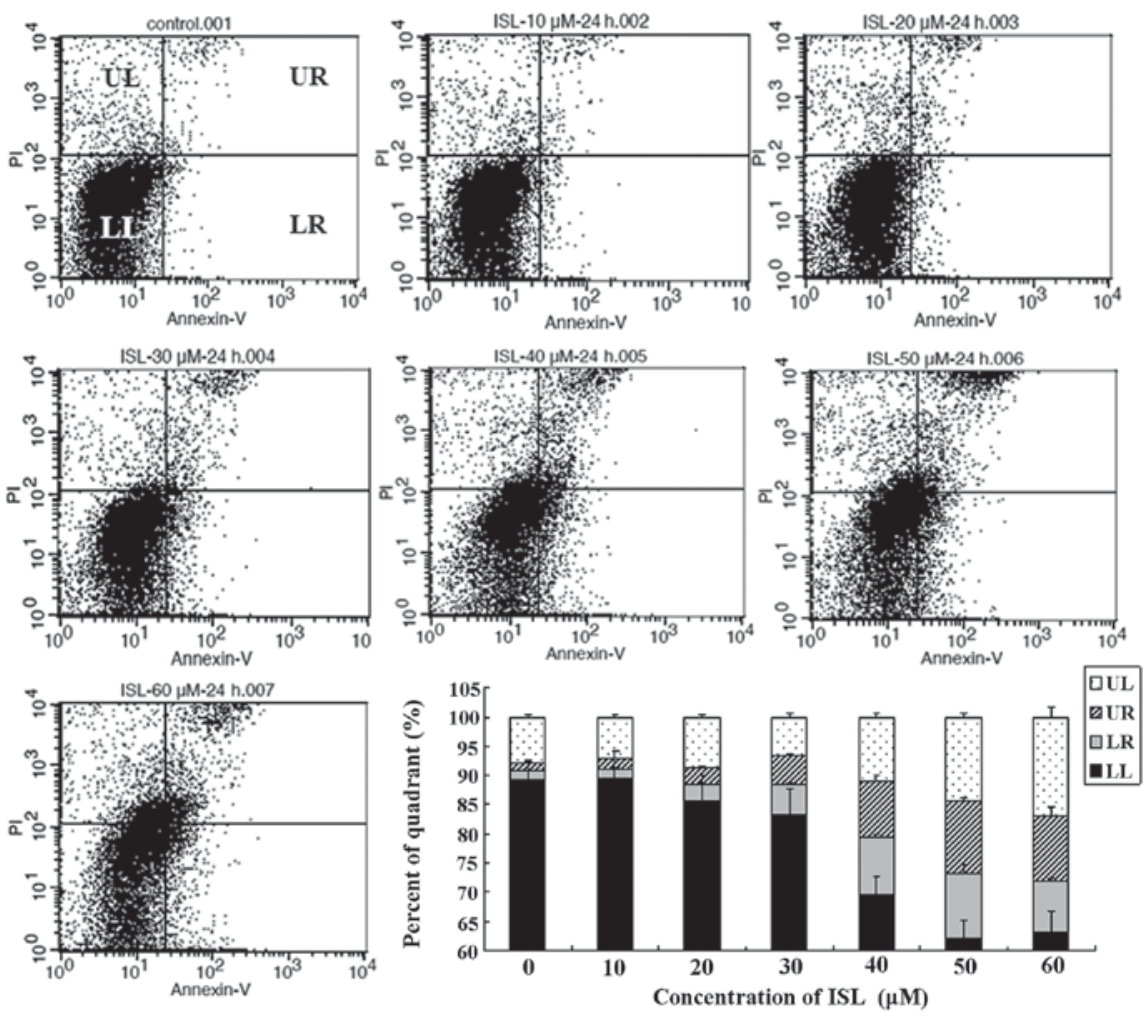

Figure 3. Apoptosis induced by isoliquiritigenin (ISL). U87 cells were treated with ISL for $24 \mathrm{~h}$ at various concentrations. The cells were trypsinized, stained with Annexin V and propidium iodide (PI) and then analyzed by flow cytometry. LL, living cells; UL, non-apoptotic necrotic cells; LR, early apoptotic cells; UR, cells undergoing secondary apoptosis and late stages of necrosis. Data are expressed as a percentage of total cell number. Each bar represents the mean $\pm \mathrm{SD}$.
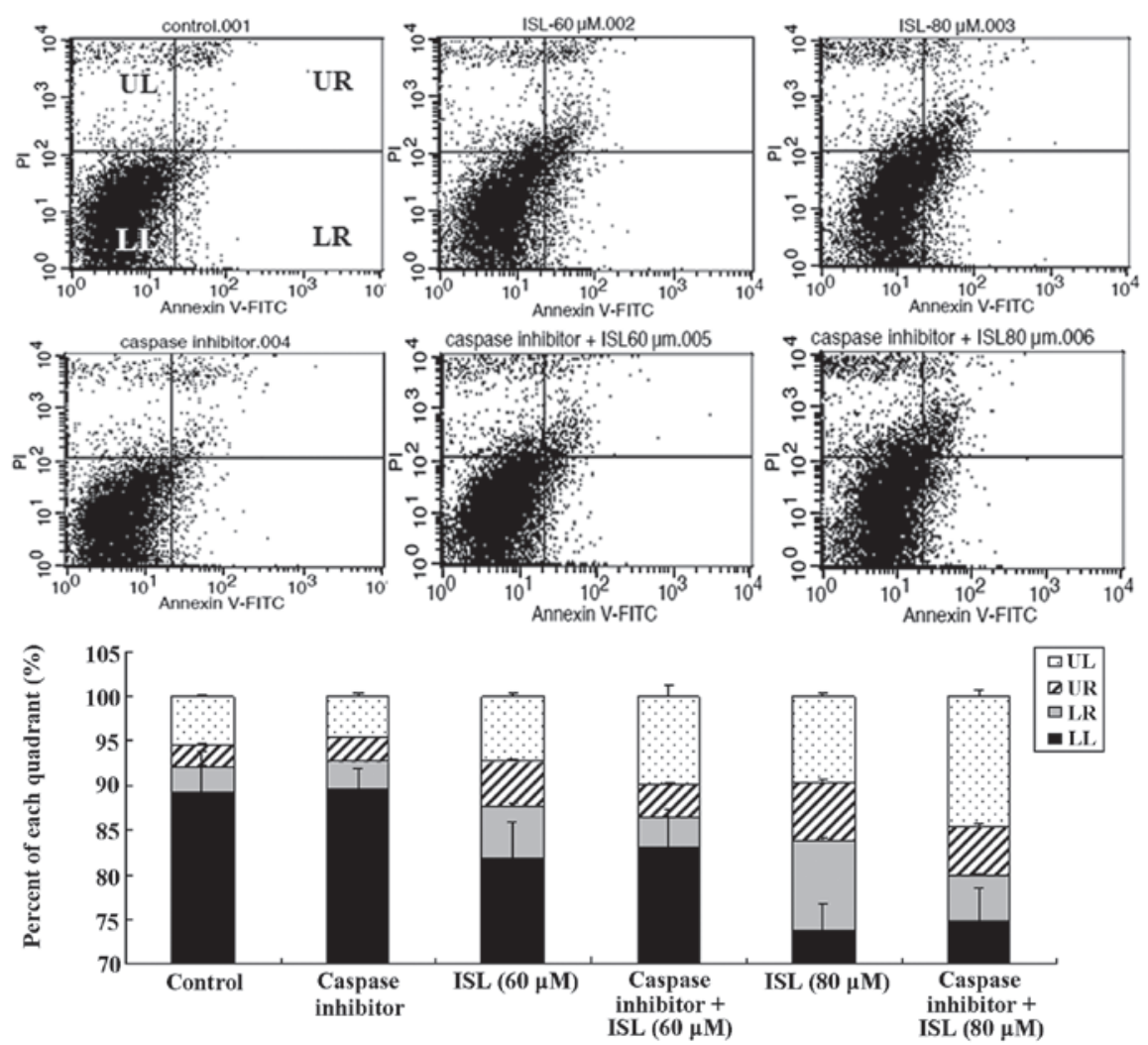

Figure 4. Caspase inhibitor Z-VAD-FMK decreases isoliquiritigenin (ISL)-induced apoptotic cell death, but not necrotic cell death in U87 glioma cells. The U87 cells were preincubated with or without $25 \mu \mathrm{mol} / 1 \mathrm{Z}$-VAD-FMK for $2 \mathrm{~h}$ and then treated with ISL for $24 \mathrm{~h}$. LL, living cells; UL, non-apoptotic necrotic cells; LR, early apoptotic cells; UR, cells undergoing secondary apoptosis and late stages of necrosis. Data are representative of three independent experiments with similar results. 

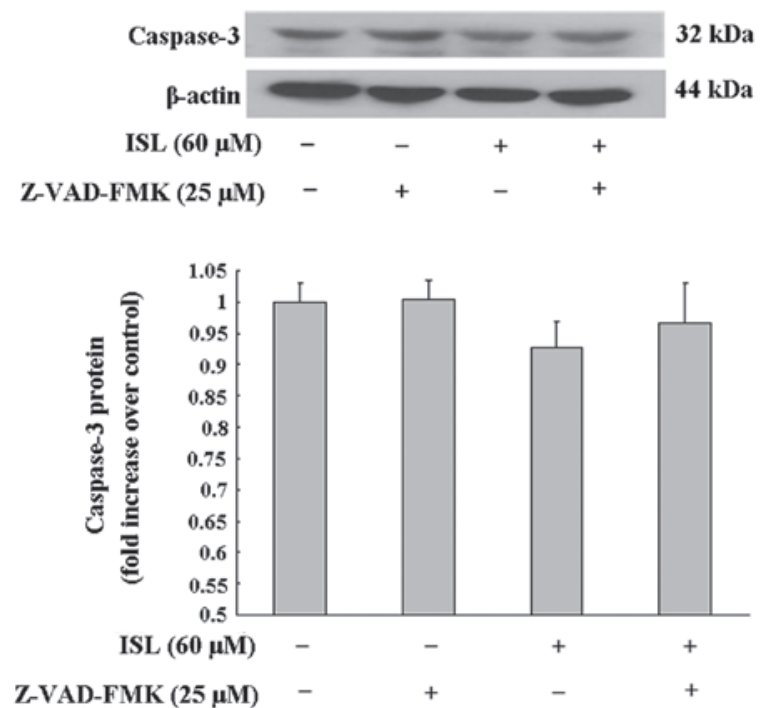

Figure 5. Effects of the pan-caspase inhibitor Z-VAD-FMK on the isoliquiritigenin (ISL)-induced caspase-3 activation in U87 glioma cells. The cells were pre-treated with or without Z-VAD-FMK $(25 \mu \mathrm{M})$ for $2 \mathrm{~h}$ and then exposed to ISL $(60 \mu \mathrm{M})$ for $24 \mathrm{~h}$. After treatment, the expression of caspase-3 was analyzed by western blot analysis as described in Materials and methods Band density data are expressed as the mean \pm SD
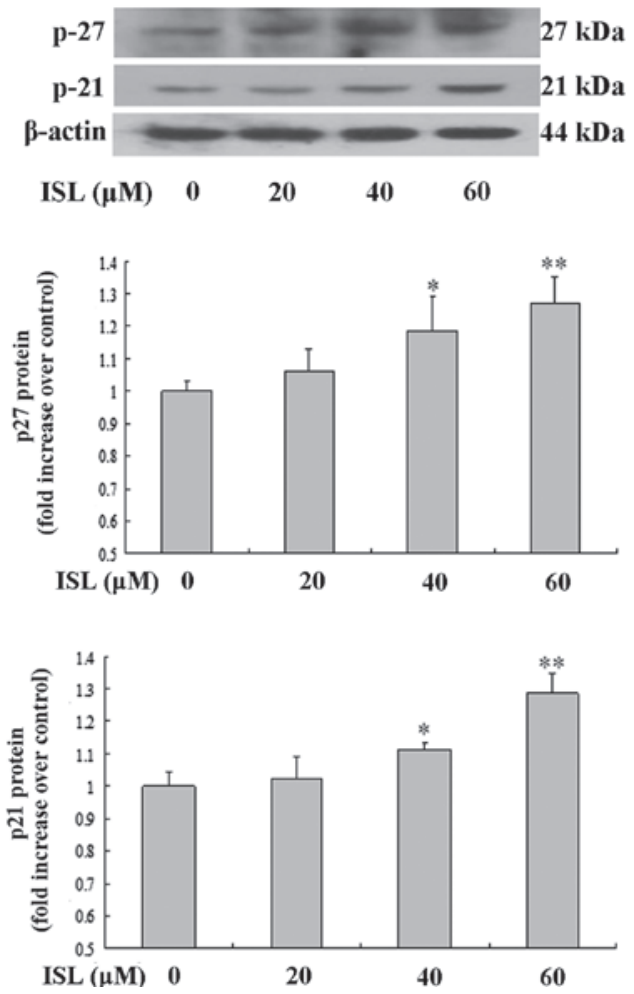

Figure 6. Effects of isoliquiritigenin (ISL) on p27 and p21 protein expression in U87 cells. U87 human glioma cells were treated with ISL $(0,20,40$ and $60 \mu \mathrm{M})$, p21 and p27 levels were determined by western blot and analyzed using Image J software. Data are the mean \pm SD of three determinations. ${ }^{*} \mathrm{P}<0.05$ compared with the control $\left(0 \mu \mathrm{M}\right.$ ISL); ${ }^{* *} \mathrm{P}<0.01$ compared with the control $(0 \mu \mathrm{M}$ ISL $)$

by western blot analysis. As shown in Fig. 6, the expression levels of the p21 and p27 proteins were upregulated as the ISL concentration increased.

\section{Discussion}

ISL has been considered as a potential therapeutic agent for the treatment of cancer, and has demonstrated antitumor efficacy in various types of cancer, including colon $(9,12)$, breast $(13,14)$, gastric $(15)$ and prostate cancer (16) and melanoma (17). However, the effects of ISL in glioma cancer cells remain unknown. In this study, we examined the effect and mechanism of ISL on human glioma cell growth. Our results revealed that ISL inhibited the growth of U87 glioma cells in a time-dependent and dose-dependent manner. Treatment of the U87 cells with ISL resulted in G2/M-phase and S-phase cell cycle arrest and induced apoptosis in a dose-dependent manner. The blockade of cell cycle progression by ISL was achieved through upregulation of the p21/WAF1 and p27 pathways. In addition, the cell death of U87 cells induced by ISL progressed via apoptotic and necrotic pathways.

The results of MTT assays revealed that ISL inhibited the proliferation of U87 cells in a time- and dose-dependent manner. However, the MTT assay system determined only the total percentage of cell death and did not discriminate between cell death occurring via apoptotic or necrotic pathways. With Annexin V/PI doubled staining using FACS for phosphatidyl serine detection, it was identified that ISL induced the death of the U87 glioma cells in apoptotic and non-apoptotic manners. We identified that the necrotic phenotype occurred in more cells than the apoptotic phenotype, therefore, necrosis may be the prevalent response to ISL treatment in U87 glioma cells. To further investigate whether the underlying mechanism of cell death induced by ISL was caspase-related or not, we blocked caspases with the pan-caspase inhibitor Z-VAD-FMK and analyzed the cell death. The results of western blot analysis revealed that caspase-3 activation was effectively blocked, however, the percentage of living cells did not increase, which suggests that ISL-induced cell death occured in a caspase-independent manner. The results of FACS also revealed that the percentage of necrotic cells increased with the increase of ISL concentration and was not altered by treatment with the pan-caspase inhibitor Z-VAD-FMK, indicating that the non-apoptotic cell death induced by ISL was predominant compared with the cell death occurring via an apoptotic pathway.

It was also identified that p21/WAF1 and p27 were upregulated by the treatment with ISL, in accordance with the results reported by others (18-20), indicating that the G2/M arrest induced by ISL is a general effect occurring in multiple cell lines. p21/WAF1 and p27 are universal inhibitors of cyclin-dependent kinases and play important roles in G2/M arrest. The upregulation of p21 and p27 may contribute to the antiproliferative activity of ISL in U87 glioma cells.

In summary, the results of our study suggest that ISL induced apoptotic and necrotic cell death of U87 glioma cells, and that ISL-induced cell death occurred in the presence of the caspase inhibitor Z-VAD-FMK. The ISL induced signaling involves the upregulation of p21/WAF1 and p27. As ISL has demonstrated evident bioactivity in cancer therapy and prevention, distinguishing the onset of ISL-induced apoptosis or necrosis using an in vitro cell culture system is likely to be important for the utilization of this compound as a potential therapeutic agent in humans. 


\section{References}

1. Lima FR, Kahn SA, Soletti RC, Biasoli D, Alves T, da Fonseca AC, et al: Glioblastoma: Therapeutic challenges, what lies ahead. Biochim Biophys Acta 1826: 338-349, 2012.

2. Ma X, Lv Y, Liu J, Wang D, Huang Q, Wang X, Li G, Xu S and Li X: Survival analysis of 205 patients with glioblastoma multiforme: clinical characteristics, treatment and prognosis in China. J Clin Neurosci 16: 1595-1598, 2009.

3. Davis ME and Stoiber AM: Glioblastoma multiforme: enhancing survival and quality of life. Clin J Oncol Nurs 15: 291-297, 2011.

4. Lin H, Liu J and Zhang Y: Developments in cancer prevention and treatment using traditional Chinese medicine. Front Med 5 : 127-133, 2011.

5. Efferth T, Li PC, Konkimalla VS and Kaina B: From traditional Chinese medicine to rational cancer therapy. Trends Mol Med 13: 353-361, 2007.

6. Wang S, Wu X, Tan M, Gong J, Tan W, Bian B, Chen M and Wang Y: Fighting fire with fire: poisonous Chinese herbal medicine for cancer therapy. J Ethnopharmacol 140: 33-45, 2012.

7. Kandaswami C, Lee LT, Lee PP, Hwang JJ, Ke FC, Huang YT and Lee MT: The antitumor activities of flavonoids. In Vivo 19: 895-909, 2005.

8. Weng CJ and Yen GC: Flavonoids, a ubiquitous dietary phenolic subclass, exert extensive in vitro anti-invasive and in vivo antimetastatic activities. Cancer Metastasis Rev 31: 323-351, 2012.

9. Lee CK, Son SH, Park KK, Park JH, Lim SS and Chung WY: Isoliquiritigenin inhibits tumor growth and protects the kidney and liver against chemotherapy-induced toxicity in a mouse xenograft model of colon carcinoma. J Pharmacol Sci 106: 444-451, 2008.

10. Orlikova B, Schnekenburger M, Zloh M, Golais F, Diederich M and Tasdemir D: Natural chalcones as dual inhibitors of HDACs and NF-кB. Oncol Rep 28: 797-805, 2012.
11. Lee YM, Jeong GS, Lim HD, An RB, Kim YC and Kim EC: Isoliquiritigenin 2-methyl ether induces growth inhibition and apoptosis in oral cancer cells via heme oxygenase-1. Toxicol In Vitro 24: 776-782, 2010.

12. Auyeung KK and Ko JK: Novel herbal flavonoids promote apoptosis but differentially induce cell cycle arrest in human colon cancer cell. Invest New Drugs 28: 1-13, 2010.

13. Lau GT, Ye L and Leung LK: The licorice flavonoid isoliquiritigenin suppresses phorbol ester-induced cyclooxygenase- 2 expression in the non-tumorigenic MCF-10A breast cell line. Planta Med 76: 780-785, 2010.

14. Ye L, Gho WM, Chan FL, Chen S and Leung LK: Dietary administration of the licorice flavonoid isoliquiritigenin deters the growth of MCF-7 cells overexpressing aromatase. Int J Cancer 124: 1028-1036, 2009.

15. Ma J, Fu NY, Pang DB, Wu WY and Xu AL: Apoptosis induced by isoliquiritigenin in human gastric cancer MGC-803 cells. Planta Med 67: 754-777, 2001.

16. Zhang X, Yeung ED, Wang J, Panzhinskiy EE, Tong C, Li W and $\mathrm{Li}$ J: Isoliquiritigenin, a natural anti-oxidant, selectively inhibits the proliferation of prostate cancer cells. Clin Exp Pharmacol Physiol 37: 841-847, 2010.

17. Iwashita K, Kobori M, Yamaki K and Tsushida T: Flavonoids inhibit cell growth and induce apoptosis in B16 melanoma 4A5 cells. Biosci Biotechnol Biochem 64: 1813-1820, 2000.

18. Hsu YL, Kuo PL, Chiang LC and Lin CC: Isoliquiritigenin inhibits the proliferation and induces the apoptosis of human non-small cell lung cancer A549 cells. Clin Exp Pharmacol Physiol 31: 414-418, 2004.

19. Li T, Satomi Y, Kotoh D, Shimada J, Baba M, Okuyama T, Nishino $\mathrm{H}$ and Kitamura N: Induction of cell cycle arrest and p21(CIP1/WAF1) expression in human lung cancer cells by isoliquiritigenin. Cancer Lett 207: 27-35, 2004.

20. Lee YM, Lim do Y, Choi HJ, Jung JL, Chung WY and Park JH: Induction of cell cycle arrest in prostate cancer cells by the dietary compound isoliquiritigenin. J Med Food 12: 8-14, 2009. 